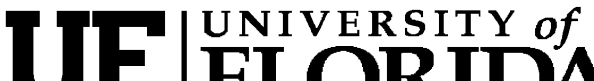 FLORIDA \\ IFAS Extension
}

\section{Pesticide Labeling: Signal Words $\mathbf{1}$}

\section{Frederick M. Fishel $^{2}$}

This document interprets signal words seen on pesticide labels and discusses the toxicity criteria used in determining the appropriate signal word for the pesticide. Examples of typical statements found on pesticide labels which convey information to the handler of the product are provided.

\section{Introduction}

When reviewing a pesticide label prior to handling the product, one may see a prominent display of one word on the front panel of the label. What does it mean to see one of the following signal words: "CAUTION," "WARNING," or "DANGER?" What does it mean if the products label has no signal word displayed? The signal word conveys a message to the products handler regarding its acute toxicity.

The signal word for a pesticide is typically determined by the results of the six acute toxicity studies performed with the product formulation. The acute oral, dermal and inhalation studies evaluate systemic toxicity by those routes of entry. The primary eye and skin irritation studies measure irritation or corrosion, while the dermal sensitization study evaluates the potential for allergic contact dermatitis. With the exception of dermal sensitization, each acute study is assigned to a toxicity category based on the study results (Table 1).

\section{Determining the precautionary labeling signal word}

When required. A signal word is required for all registered pesticide products unless the pesticide product meets the criteria of Toxicity Category IV by all routes of exposure. If a pesticide manufacturer desires its label to list a signal word in this case, it must be "CAUTION."

Determining the signal word. The signal word is determined by the most severe toxicity category assigned to the five acute toxicity studies seen in Table 1 or by the presence of methanol in concentrations of $4 \%$ or more. Table 2 lists the appropriate signal word based upon toxicity category. Examples of appropriate signal words based upon toxicity studies are provided in Table 3 . Typical statements seen on pesticide labels for acute oral, dermal, and inhalation toxicity are shown in Tables 4, 5 and 6, respectively. Typical statements seen on pesticide labels for products which potentially cause primary eye irritation are shown in Table 7 and

1. This document is PI-100, one of a series of the Pesticide Information Office, Florida Cooperative Extension Service, Institute of Food and Agricultural Sciences, University of Florida. Original publication date February 2006. Visit the EDIS Web Site at http://edis.ifas.ufl.edu.

2. Frederick M. Fishel, associate professor, Agronomy Department, and Director, Pesticide Information Office; Florida Cooperative Extension Service, Institute of Food and Agricultural Sciences, University of Florida, Gainesville, FL 32611.

Use pesticides safely. Read and follow directions on the manufacturer's label.

The Institute of Food and Agricultural Sciences (IFAS) is an Equal Opportunity Institution authorized to provide research, educational information and other services only to individuals and institutions that function with non-discrimination with respect to race, creed, color, religion, age, disability, sex, sexual orientation, marital status, national origin, political opinions or affiliations. U.S. Department of Agriculture, Cooperative Extension Service, University of Florida, IFAS, Florida A. \& M. University Cooperative Extension Program, and Boards of County Commissioners Cooperating. Larry Arrington, Dean 
statements for products which potentially cause primary skin irritation are shown in Table 8 . Table 9 lists typical statements for dermal sensitization.

Location and prominence. The signal word is required to appear on the front panel of the label, and EPA requests pesticide manufacturers to place it on a separate line from the required Child Hazard Warning statement (Keep Out of Reach of Children). The signal word is also required on any supplemental label intended to accompany the product in distribution or sale.

Related information. Because of the potential for confusion, EPA historically has not approved labels containing the terms, "caution," "warning," or "danger," except as the signal word for that label. For example: "CAUTION: Wash hands before eating, or smoking" on a label with the signal of "CAUTION."

\section{POISON - skull and crossbones symbol}

When required. The word "POISON" and the skull and crossbones symbol are required for products classified as toxicity category I for acute oral, acute dermal, or acute inhalation toxicity studies. If the inert ingredient, methanol, is present at $4 \%$ or more in the product, EPA suggests that the manufacturer post the skull and crossbones symbol on the label. Examples are shown in Table 3.

Location and prominence. If required, the word "POISON" and the skull and crossbones symbol must appear in immediate proximity to each other. The word "POISON" must appear in red on a background of a distinctly contrasting color. In addition, EPA requests that the "POISON" and the skull and crossbones symbol appear near the signal word "DANGER" (Figure 1).

\section{Additional Information}

Dean, T.W. 2003. Pesticide applicator update: choosing suitable personal protective equipment. UF/IFAS EDIS Document PI-28. http://edis.ifas.ufl.edu/PI061.

\section{DANGER/ PELIGRO}

Figure 1. "POISON" and the skull-and-crossbones symbol shoould appear near the signal word "DANGER".

Fishel, F.M. 2005. Interpreting pesticide label wording. UF/IFAS EDIS Document PI-34. http://edis.ifas.ufl.edu/PI071.

Fishel, F.M. 2005. Respirators for pesticide applications. UF/IFAS EDIS Document PI-77. http://edis.ifas.ufl.edu/PI114.

Nesheim, O.N., F.M. Fishel and M. Mossler. 2005. Toxicity of pesticides. UF/IFAS EDIS Document PI-13. http://edis.ifas.ufl.edu/PI008. 
Table 1. Toxicity categories.

\begin{tabular}{|c|c|c|c|c|}
\hline Study & Category I & Category II & Category III & Category IV \\
\hline Acute oral & $\begin{array}{l}\text { Up to and including } 50 \\
\mathrm{mg} / \mathrm{kg}\end{array}$ & $\begin{array}{l}>50 \text { through } 500 \\
\mathrm{mg} / \mathrm{kg}\end{array}$ & $\begin{array}{l}>500 \text { through } 5,000 \\
\mathrm{mg} / \mathrm{kg}\end{array}$ & $>5,000 \mathrm{mg} / \mathrm{kg}$ \\
\hline Acute dermal & $\begin{array}{l}\text { Up to and including } \\
200 \mathrm{mg} / \mathrm{kg}\end{array}$ & $\begin{array}{l}>200 \text { through } 2,000 \\
\mathrm{mg} / \mathrm{kg}\end{array}$ & $\begin{array}{l}>2,000 \text { through } \\
5,000 \mathrm{mg} / \mathrm{kg}\end{array}$ & $>5,000 \mathrm{mg} / \mathrm{kg}$ \\
\hline $\begin{array}{l}\text { Acute } \\
\text { inhalation }^{*}\end{array}$ & $\begin{array}{l}\text { Up to and including } \\
0.05 \mathrm{mg} / \mathrm{liter}\end{array}$ & $\begin{array}{l}>0.05 \text { through } 0.5 \\
\mathrm{mg} / \text { /iter }\end{array}$ & $\begin{array}{l}>0.5 \text { through } 2 \\
\text { mg/liter }\end{array}$ & $>2$ mg/liter \\
\hline $\begin{array}{l}\text { Primary eye } \\
\text { irritation }\end{array}$ & $\begin{array}{l}\text { Corrosive (irreversible } \\
\text { destruction of ocular } \\
\text { tissue) or corneal } \\
\text { involvement or } \\
\text { irritation persisting for } \\
\text { more than } 21 \text { days }\end{array}$ & $\begin{array}{l}\text { Corneal } \\
\text { involvement or } \\
\text { other eye irritation } \\
\text { clearing in 8-21 days }\end{array}$ & $\begin{array}{l}\text { Corneal } \\
\text { involvement or } \\
\text { other eye irritation } \\
\text { clearing in } 7 \text { days or } \\
\text { less }\end{array}$ & $\begin{array}{l}\text { Minimal effects } \\
\text { clearing in less than } \\
24 \text { hours }\end{array}$ \\
\hline $\begin{array}{l}\text { Primary skin } \\
\text { irritation }\end{array}$ & $\begin{array}{l}\text { Corrosive (tissue } \\
\text { destruction into the } \\
\text { dermis and/or scarring) }\end{array}$ & $\begin{array}{l}\text { Severe irritation at } \\
72 \text { hours (severe } \\
\text { erythema or edema) }\end{array}$ & $\begin{array}{l}\text { Moderate irritation } \\
\text { at } 72 \text { hours } \\
\text { (moderate } \\
\text { erythema) }\end{array}$ & $\begin{array}{l}\text { Mild or slight } \\
\text { irritation at } 72 \text { hours } \\
\text { (no irritation or slight } \\
\text { erythema) }\end{array}$ \\
\hline
\end{tabular}

Table 2. Signal word as determined by toxicity category.

\begin{tabular}{|c|c||}
\hline \hline Toxicity category & Signal word \\
\hline I & DANGER \\
\hline II & WARNING \\
\hline III & CAUTION \\
\hline IV & None required \\
\hline \hline
\end{tabular}

Table 3. Examples of signal words based upon toxicity studies.

\begin{tabular}{|c|c|c|c|c|c|}
\hline Type of study & Product A & Product B & Product C & Product D & Product E \\
\hline Acute oral & III & IV & $1^{*}$ & III & II \\
\hline Acute dermal & IV & III & III & IV & II \\
\hline Acute inhalation & III & IV & III & III & II \\
\hline Primary eye & III & II & 1 & $\mathrm{I}$ & II \\
\hline Primary skin & IV & IV & II & IV & II \\
\hline $\begin{array}{l}\text { Contains } \geq 4 \% \\
\text { methanol }\end{array}$ & No & No & No & No & $\mathrm{Yes}^{* *}$ \\
\hline Signal word & CAUTION & WARNING & DANGER & DANGER & DANGER \\
\hline \multicolumn{6}{|c|}{$\begin{array}{l}\text { *Product C must also bear the skull and crossbones symbol in close proximity to the word "POISON" which } \\
\text { must appear in red on a contrasting background due to acute oral toxicity. } \\
\text { ** Product E must also bear the skull and crossbones symbol in close proximity to the word "POISON" which } \\
\text { must appear in red on a contrasting background due to its formulation containing at least } 4 \% \text { methanol. }\end{array}$} \\
\hline
\end{tabular}


Table 4. Typical statements for acute oral toxicity.

\begin{tabular}{||c|l|l||}
\hline \hline Toxicity category & Signal word & Statements \\
\hline I & $\begin{array}{l}\text { DANGER-POISON Skull } \\
\text { and crossbones required }\end{array}$ & $\begin{array}{l}\text { Fatal if swallowed. Wash thoroughly with soap and } \\
\text { water after handling and before eating, drinking, } \\
\text { chewing gum, or using tobacco. }\end{array}$ \\
\hline II & WARNING & $\begin{array}{l}\text { May be fatal if swallowed. Wash thoroughly with soap } \\
\text { and water after handling and before eating, drinking, } \\
\text { chewing gum, or using tobacco. }\end{array}$ \\
\hline III & CAUTION & $\begin{array}{l}\text { Harmful if swallowed. Wash thoroughly with soap and } \\
\text { water after handling and before eating, drinking, } \\
\text { chewing gum, or using tobacco. }\end{array}$ \\
\hline IV & CAUTION (optional) & $\begin{array}{l}\text { No statements are required. However, manufacturers } \\
\text { may choose to use category III labeling. }\end{array}$ \\
\hline $\begin{array}{l}\text { *For products containing } \\
\text { statement should be added to the label: "Methanol may cause blindness." }\end{array}$ \\
\hline
\end{tabular}

Table 5. Typical statements for acute dermal toxicity.

\begin{tabular}{||c|l|l||}
\hline \hline Toxicity category & Signal word & Statements \\
\hline I & $\begin{array}{c}\text { DANGER-POISON Skull } \\
\text { and crossbones required }\end{array}$ & $\begin{array}{l}\text { Fatal if absorbed through skin. Do not get in eyes, on } \\
\text { skin, or on clothing. Wash thoroughly with soap and } \\
\text { water after handling and before eating, drinking, } \\
\text { chewing gum, or using tobacco. Wear (appropriate } \\
\text { protective clothing listed here). Remove and wash } \\
\text { contaminated clothing before reuse. }\end{array}$ \\
\hline II & WARNING & $\begin{array}{l}\text { May be fatal if absorbed through skin. Do not get in } \\
\text { eyes, on skin, or on clothing. Wash thoroughly with } \\
\text { soap and water after handling and before eating, } \\
\text { drinking, chewing gum, or using tobacco. Wear } \\
\text { (appropriate protective clothing listed here). Remove } \\
\text { and wash contaminated clothing before reuse. }\end{array}$ \\
\hline III & CAUTION & $\begin{array}{l}\text { Harmful if absorbed through skin. Avoid contact with } \\
\text { skin, eyes or clothing. Wash thoroughly with soap and } \\
\text { water after handling and before eating, drinking, } \\
\text { chewing gum, or using tobacco. Wear (appropriate } \\
\text { protective clothing listed here). Remove and wash } \\
\text { contaminated clothing before reuse. }\end{array}$ \\
\hline IV & $\begin{array}{l}\text { No statements are required. However, manufacturers } \\
\text { may choose to use category III labeling. }\end{array}$ \\
\hline
\end{tabular}

Table 6. Typical statements for acute inhalation toxicity.

\begin{tabular}{||c|l|l||}
\hline \hline Toxicity category & Signal word & Statements \\
\hline $\mathrm{I}$ & $\begin{array}{l}\text { DANGER-POISON Skull } \\
\text { and crossbones required }\end{array}$ & $\begin{array}{l}\text { Fatal if inhaled. Do not breathe (dust, vapor, or spray } \\
\text { mist listed here). Wear (appropriate respiratory } \\
\text { protection listed here). Remove and wash } \\
\text { contaminated clothing before reuse. }\end{array}$ \\
\hline \hline
\end{tabular}


Table 6. Typical statements for acute inhalation toxicity.

\begin{tabular}{||c|l|l||}
\hline \hline Toxicity category & Signal word & Statements \\
\hline II & WARNING & $\begin{array}{l}\text { May be fatal if inhaled. Do not breathe (dust, vapor, or } \\
\text { spray mist listed here). Wear (appropriate respiratory } \\
\text { protection listed here). Remove and wash } \\
\text { contaminated clothing before reuse. }\end{array}$ \\
\hline III & CAUTION & $\begin{array}{l}\text { Harmful if inhaled. Avoid breathing (dust, vapor, or } \\
\text { spray mist listed here). Remove and wash } \\
\text { contaminated clothing before reuse. }\end{array}$ \\
\hline IV & CAUTION (optional) & $\begin{array}{l}\text { No statements are required. However, manufacturers } \\
\text { may choose to use category III labeling. }\end{array}$ \\
\hline \hline
\end{tabular}

Table 7. Typical statements for primary eye irritation.

\begin{tabular}{|c|c|c|}
\hline Toxicity category & Signal word & Statements \\
\hline 1 & DANGER & $\begin{array}{l}\text { Corrosive. }{ }^{*} \text { Causes irreversible eye damage. Do not } \\
\text { get in eyes or on clothing. Wear (appropriate } \\
\text { protective eyewear such as goggles, face shield, or } \\
\text { safety glasses listed here). Wash thoroughly with } \\
\text { soap and water after handling and before eating, } \\
\text { drinking, chewing gum, or using tobacco. Remove } \\
\text { and wash contaminated clothing before reuse. }\end{array}$ \\
\hline II & WARNING & $\begin{array}{l}\text { Causes substantial but temporary eye injury. Do not } \\
\text { get in eyes or on clothing. Wear (appropriate } \\
\text { protective eyewear such as goggles, face shield, or } \\
\text { safety glasses listed here). Wash thoroughly with } \\
\text { soap and water after handling and before eating, } \\
\text { drinking, chewing gum, or using tobacco. Remove } \\
\text { and wash contaminated clothing before reuse. }\end{array}$ \\
\hline III & CAUTION & $\begin{array}{l}\text { Causes moderate eye irritation. Avoid contact with } \\
\text { eyes or clothing. Wear (specify protective eyewear, if } \\
\text { appropriate, here). Wash thoroughly with soap and } \\
\text { water after handling and before eating, drinking, } \\
\text { chewing gum, or using tobacco. }\end{array}$ \\
\hline IV & CAUTION (optional) & $\begin{array}{l}\text { No statements are required. However, manufacturers } \\
\text { may choose to use category III labeling. }\end{array}$ \\
\hline
\end{tabular}

Table 8. Typical statements for primary skin irritation.

\begin{tabular}{||c|l|l||}
\hline \hline Toxicity category & Signal word & Statements \\
\hline I & DANGER & $\begin{array}{l}\text { Corrosive. Causes skin burns. Do not get in eyes, on } \\
\text { skin, or on clothing. Wear (appropriate protective } \\
\text { clothing and gloves listed here). Wash thoroughly with } \\
\text { soap and water after handling and before eating, } \\
\text { drinking, chewing gum, or using tobacco. Remove } \\
\text { and wash contaminated clothing before reuse. }\end{array}$ \\
\hline
\end{tabular}


Table 8. Typical statements for primary skin irritation.

\begin{tabular}{||c|l|l||}
\hline \hline Toxicity category & Signal word & Statements \\
\hline II & WARNING & $\begin{array}{l}\text { Causes skin irritation. Do not get on skin or on } \\
\text { clothing. Wear (appropriate protective clothing and } \\
\text { gloves listed here). Wash thoroughly with soap and } \\
\text { water after handling and before eating, drinking, } \\
\text { chewing gum, or using tobacco. Remove and wash } \\
\text { contaminated clothing before reuse. }\end{array}$ \\
\hline III & CAUTION & $\begin{array}{l}\text { Avoid contact with skin or clothing. Wash thoroughly } \\
\text { with soap and water after handling and before eating, } \\
\text { drinking, chewing gum, or using tobacco. Wear } \\
\text { (appropriate protective clothing and gloves listed } \\
\text { here). }\end{array}$ \\
\hline IV & CAUTION (optional) & $\begin{array}{l}\text { No statements are required. However, manufacturers } \\
\text { may choose to use category III labeling. }\end{array}$ \\
\hline \hline
\end{tabular}

Table 9. Typical statements for dermal sensitization.

\begin{tabular}{|c|c|}
\hline Study results & Statement \\
\hline Product is a sensitizer or is positive for sensitization. & $\begin{array}{l}\text { Prolonged or frequently repeated skin contact may } \\
\text { cause allergic reactions in some individuals. }\end{array}$ \\
\hline $\begin{array}{l}\text { Product is not a sensitizer or is negative for } \\
\text { sensitization. }\end{array}$ & No labeling is required for this result. \\
\hline
\end{tabular}

